\title{
On a class of spiral-like functions with respect to a boundary point related to subordination
}

\author{
Maisarah Haji Mohd and Maslina Darus ${ }^{*}$ \\ Dedicated to Professor Hari M Srivastava
}

"Correspondence: maslina@ukm.my School of Mathematical Sciences, Faculty of Science and Technology, Universiti Kebangsaan Malaysia, Bangi, Selangor D Ehsan 43600, Malaysia

\section{Abstract}

For $\mu \in \mathbb{C}, \varphi$ a starlike univalent function, the class of functions $f$ that are spiral-like with respect to a boundary point satisfying the subordination

$$
\frac{2}{\mu} \frac{z f^{\prime}(z)}{f(z)}+\frac{1+z}{1-z} \prec \varphi(z), \quad z \in \mathbb{D},
$$

is investigated. The integral representation, growth and distortion theorem are proved by relating these functions with $\mathrm{Ma}$ and Minda starlike functions. Some earlier results are shown to be a special case of the results obtained.

MSC: $30 \mathrm{C} 80 ; 30 \mathrm{C} 45$

Keywords: subordination; starlike with respect to boundary point; spiral-like with respect to a boundary point

\section{Introduction and motivation}

Let $\mathbb{D}=\{z:|z|<1\}$ be an open unit disk of the complex plane $\mathbb{C}$ and let $\mathcal{A}$ be a class of analytic functions $f$ normalized by $f(0)=0$ and $f^{\prime}(0)=1$. Let $w_{0}$ be an interior or a boundary point of a set $\mathcal{D}$ in $\mathbb{C}$. The set $\mathcal{D}$ is starlike with respect to $w_{0}$ if the line segment joining $w_{0}$ to every other point in $\mathcal{D}$ lies in the interior of $\mathcal{D}$. If a function $f \in \mathcal{A}$ maps $\mathbb{D}$ onto a starlike domain with respect to origin, then $f$ is a starlike function. The class of starlike functions with respect to origin is denoted by $\mathcal{S}^{*}$. Analytically,

$$
\mathcal{S}^{\prime \prime}:=\left\{f \in \mathcal{A}: \operatorname{Re} \frac{z f^{\prime}(z)}{f(z)}>0\right\}
$$

Robertson [1] took a leap forward with the characterization of the class $\mathcal{S}^{\prime \prime}$ and defined the class $\mathcal{S}_{b}^{*}$ of starlike functions with respect to a boundary point. Geometrically, it is the characterization of a function $f \in \mathcal{S}_{b}=\left\{f(z)=1+d_{1} z+d_{2} z^{2}+\cdots \mid f\right.$ univalent $\}$ such that $f(\mathbb{D})$ is starlike with respect to the boundary point $f(1):=\lim _{r \rightarrow 1^{-}} f(r)=0$ and lies in a half-plane. The analytic description given by Robertson was

$$
\mathcal{S}_{b}^{*}:=\left\{f \in \mathcal{S}_{b}: \operatorname{Re}\left(2 \frac{z f^{\prime}(z)}{f(z)}+\frac{1+z}{1-z}\right)>0\right\} .
$$

@ 2013 Haji Mohd and Darus; licensee Springer. This is an Open Access article distributed under the terms of the Creative Commons Attribution License (http://creativecommons.org/licenses/by/2.0), which permits unrestricted use, distribution, and reproduction in any medium, provided the original work is properly cited. 
This was partially proved in [1]. It was only in 1984 that the characterization was validated by Lyzzaik [2]. Todorov [3] associated this class with a functional $f(z) /(1-z)$ and obtained a structured formula and coefficient estimates in the year 1986. Later, Silverman and Silvia [4] gave a full description of the class of univalent functions on $\mathbb{D}$, the image of which is star-shaped, with respect to a boundary point. Since then, this class of starlike functions with respect to a boundary point has gained notable interest among geometric function theorist and also other researchers. Among them, Abdullah et al. [5] studied the properties of functions in this class. The distortion results for starlike functions with respect to a boundary point were obtained in [6,7]. The dynamical characterizations of functions starlike with respect to a boundary point can be found in [8]. In the year 2001, Lecko [9] gave another representation of starlike functions with respect to a boundary point. Also, Lecko and Lyzzaik obtained different characterizations of this class in [10].

Following the studies on the class of starlike functions, many authors extensively studied the class of spiral-like functions. For recent work on the class of spiral-like functions, see [11]. Later, there was interest towards the class of spiral-like functions with respect to a boundary point. See [12-15]. Aharonov et al. [16] gave a comprehensive definition for spiral-shaped domains with respect to a boundary point.

Definition 1.1 A simply connected domain $\Omega \subset \mathbb{C}, 0 \in \partial \Omega$, is called a spiral-shaped domain with respect to a boundary point if there is a number $\mu \in \mathbb{C}$ with $\operatorname{Re} \mu>0$ such that, for any point $\omega \in \Omega$, the curve $e^{-t \mu} \omega, t \geq 0$, is contained in $\Omega$.

It was also showed in [16] (see also [17]) that each spiral-like function with respect to a boundary point is a complex power of starlike function with respect to a boundary point. In particular, if $\mu \in \mathbb{R}$ in Definition 1.1, then $\Omega$ is called a star-shaped domain with respect to a boundary point. The following was proved in the same.

Theorem 1.1 Let $f$ be an analytic function with $f(0)=1, f(1)=0$, and let it be a spiral-like function with respect to a boundary point. Then there exists a number $\mu \in \Omega:=\{\lambda \in \mathbb{C}$ : $|\lambda-1| \leq 1, \lambda \neq 0\}$ such that

$$
\operatorname{Re}\left(\frac{2}{\mu} \frac{z f^{\prime}(z)}{f(z)}+\frac{1+z}{1-z}\right)>0
$$

Conversely, if $f$ is a univalent function with $f(0)=1$ and $f(1)=0$ satisfies (1.1) for some $\mu \in \Omega$, then $f$ is a spiral-like function with respect to a boundary point.

Elin [18] then considered the class of spiral-like functions of order $\beta(0<\beta \leq 1)$ with respect to a boundary point and obtained interesting results including the distortion and covering theorems.

On the other hand, Ma and Minda [19] gave a unified presentation of the class starlike using the method of subordination. For two functions $h$ and $g$ in $\mathcal{A}$, the function $h$ is subordinate to $g$, written

$$
h(z) \prec g(z), \quad z \in \mathbb{D},
$$

if there exists a function $w \in \mathcal{A}$, with $w(0)=0$ and $|w(z)|<1$, such that $h(z)=g(w(z))$. In particular, if the function $g$ is univalent in $\mathbb{D}$, then $h(z) \prec g(z)$ is equivalent to $h(0)=g(0)$ 
and $h(\mathbb{D}) \subset g(\mathbb{D})$. A function $h \in \mathcal{A}$ is starlike if $z h^{\prime}(z) / h(z)$ is subordinated to $(1+z) /(1-z)$. Ma and Minda [19] introduced the class

$$
\mathcal{S}^{*}(\varphi)=\left\{h \in \mathcal{A}: \frac{z h^{\prime}(z)}{h(z)} \prec \varphi(z)\right\},
$$

where $\varphi$ is an analytic function with a positive real part in $\mathbb{D}, \varphi(\mathbb{D})$ is symmetric with respect to the real axis and starlike with respect to $\varphi(0)=1$ and $\varphi^{\prime}(0)>0$. A function $f \in \mathcal{S}^{*}(\varphi)$ is called Ma and Minda starlike (with respect to $\varphi$ ). The class $\mathcal{S}^{*}(\beta)$ consisting of starlike functions of order $\beta, 0 \leq \beta<1$ and the class $\mathcal{S}^{*}(A, B)$ of Janowski starlike functions are special cases of $\mathcal{S}^{*}(\varphi)$ when $\varphi(z):=(1+(1-2 \beta) z) /(1-z)$ and $\varphi(z):=(1+A z) /(1+B z)$ for $-1 \leq B<A \leq 1$, respectively.

In the same direction and motivated mainly by [18] and [19], we consider the following class.

Definition 1.2 Let $f \in \mathcal{S}_{b}, f(0)=1$ and $\mu \in \Omega:=\{\lambda \in \mathbb{C}:|\lambda-1| \leq 1, \lambda \neq 0\}$. Also, let $\varphi$ be an analytic function with a positive real part $\mathbb{D}$, let $\varphi(\mathbb{D})$ be symmetric with respect to the real axis and starlike with respect to $\varphi(0)=1$ and $\varphi^{\prime}(0)>0$. The function $f \in \mathcal{S}_{b}^{\prime \prime}(\mu, \varphi)$ if the subordination

$$
\frac{2}{\mu} \frac{z f^{\prime}(z)}{f(z)}+\frac{1+z}{1-z} \prec \varphi(z), \quad z \in \mathbb{D},
$$

holds.

For $\varphi(z)=(1+A z) /(1+B z)(-1 \leq B<A \leq 1)$, denote the class $\mathcal{S}_{b}^{*}(\mu, \varphi)$ by $\mathcal{S}_{b}^{*}(\mu, A, B)$. For $0 \leq \beta<1, A=1-2 \beta$ and $B=-1$, denote $\mathcal{S}_{b}^{*}(\mu, A, B)$ by $\mathcal{S}_{b}^{*}(\mu, \beta)$.

The class $\mathcal{S}_{b}^{\prime \prime}(\mu, \varphi)$ defined by subordination is investigated to obtain representation, estimates for $f$ and $f^{\prime}$ and subordination conditions. We obtained some interesting result in a wider context and our approach is mainly based on [19].

\section{Representation for the class $\mathcal{S}_{b}^{*}(\mu, \varphi)$}

The following result provides an integral representation of functions belonging to the class $\mathcal{S}_{b}^{*}(\mu, \varphi)$.

Theorem 2.1 The function $f \in \mathcal{S}_{b}^{*}(\mu, \varphi)$ if and only if there exists $p$ satisfying $p \prec \varphi$ such that

$$
f(z)=(1-z)^{\mu} \exp \left(\frac{\mu}{2} \int_{0}^{z} \frac{p(\zeta)-1}{\zeta} d \zeta\right) .
$$

Proof Let $f \in \mathcal{S}_{b}^{*}(\mu, \varphi)$. Then define $p: \mathbb{D} \rightarrow \mathbb{C}$ by

$$
p(z)=\frac{2}{\mu} \frac{z f^{\prime}(z)}{f(z)}+\frac{1+z}{1-z} .
$$

Then $f \in \mathcal{S}_{b}^{*}(\mu, \varphi)$ implies that $p \prec \varphi$. Rewriting the above equation as

$$
\frac{2}{\mu} \frac{f^{\prime}(z)}{f(z)}+\frac{2}{1-z}=\frac{p(z)-1}{z}
$$


and integrating from 0 to $z$, it follows that

$$
\log \left(\frac{f(z)^{\frac{2}{\mu}}}{(1-z)^{2}}\right)=\int_{0}^{z} \frac{p(\zeta)-1}{\zeta} d \zeta
$$

An exponentiation gives

$$
f(z)^{\frac{2}{\mu}}=(1-z)^{2} \exp \left(\int_{0}^{z} \frac{p(\zeta)-1}{\zeta} d \zeta\right)
$$

The desired result follows from this. The converse follows easily.

\section{Estimates for $f$ and $f^{\prime}$ in the class $\mathcal{S}_{b}^{*}(\mu, \varphi)$}

Theorem 3.1 Let $h_{\varphi}$ be an analytic function with $h_{\varphi}(0)=0, h_{\varphi}^{\prime}(0)=1$ satisfying the equation $z h_{\varphi}^{\prime}(z) / h_{\varphi}(z)=\varphi(z)$. Iff $\in \mathcal{S}_{b}^{*}(\mu, \varphi)$, then

$$
\frac{-h_{\varphi}(-r)}{r}|1-z|^{2} \leq\left|f(z)^{\frac{2}{\mu}}\right| \leq \frac{h_{\varphi}(r)}{r}|1-z|^{2}, \quad|z|=r .
$$

Proof Define the function $h \in \mathcal{A}$ by

$$
h(z)=\frac{z}{(1-z)^{2}} f(z)^{\frac{2}{\mu}}, \quad z \in \mathbb{D} .
$$

Since $f$ is univalent and $f(1):=\lim _{r \rightarrow 1^{-}} f(r)=0$, it is clear that $f(z) \neq 0$ in $\mathbb{D}$. Therefore, the function $h$ is well defined and analytic in $\mathbb{D}$. A computation shows that

$$
\frac{z h^{\prime}(z)}{h(z)}=\frac{2}{\mu} \frac{z f^{\prime}(z)}{f(z)}+\frac{1+z}{1-z} .
$$

Hence we have the relation $f \in \mathcal{S}_{b}^{*}(\mu, \varphi)$ if and only if $h \in \mathcal{S}^{*}(\varphi)$. Ma and Minda [19, Corollary $\left.1^{\prime}\right]$ have shown that for $h \in \mathcal{S}^{*}(\varphi)$,

$$
-h_{\varphi}(-r) \leq|h(z)| \leq h_{\varphi}(r), \quad|z|=r .
$$

Using this inequality for $h$ in (3.2) gives

$$
-h_{\varphi}(-r) \leq\left|\frac{z}{(1-z)^{2}} f(z)^{\frac{2}{\mu}}\right| \leq h_{\varphi}(r), \quad|z|=r
$$

and hence the desired result follows.

If $\mathcal{S}_{b}^{*}(\mu, A, B)$ and hence

$$
h_{\varphi}(z)= \begin{cases}z(1+B z)^{\frac{A-B}{B}}, & B \neq 0, \\ z \exp (A z), & B=0,\end{cases}
$$

then

$$
\begin{aligned}
& |1-z|^{2}(1-B r)^{\frac{A-B}{B}} \leq\left|f(z)^{\frac{2}{\mu}}\right| \leq|1-z|^{2}(1+B r)^{\frac{A-B}{B}} \quad \text { for } B \neq 0, \\
& |1-z|^{2} \exp (-A r) \leq\left|f(z)^{\frac{2}{\mu}}\right| \leq|1-z|^{2} \exp (A r) \quad \text { for } B=0 .
\end{aligned}
$$




$$
\begin{aligned}
& \text { If } \mathcal{S}_{b}^{*}(\mu, \beta) \text { and } \\
& \qquad h_{\varphi}(z)=\frac{z}{(1-z)^{2-2 \beta}},
\end{aligned}
$$

then

$$
\frac{|1-z|^{2}}{(1+r)^{2-2 \beta}} \leq\left|f(z)^{\frac{2}{\mu}}\right| \leq \frac{|1-z|^{2}}{(1-r)^{2-2 \beta}} .
$$

In particular, for $0 \neq \mu \in \mathbb{R}$, the inequality reduces to the following inequality [18]:

$$
\frac{|1-z|^{\mu}}{(1+r)^{\mu(1-\beta)}} \leq|f(z)| \leq \frac{|1-z|^{\mu}}{(1-r)^{\mu(1-\beta)}} .
$$

Theorem 3.2 Let $\varphi(z)=z h_{\varphi}^{\prime}(z) / h_{\varphi}(z)$ and $f \in \mathcal{S}_{b}^{\prime \prime}(\mu, \varphi)$. Then, for $|z|=r$,

$$
\left|\arg \frac{f(z)^{\frac{1}{\mu}}}{(1-z)}\right| \leq \frac{1}{2} \max _{|z|=r} \arg \frac{h_{\varphi}(z)}{z} .
$$

For $0 \neq \mu \in \mathbb{R}$,

$$
\left|\arg \frac{f(z)}{(1-z)^{\mu}}\right| \leq \frac{|\mu|}{2} \max _{|z|=r} \arg \frac{h_{\varphi}(z)}{z} .
$$

Proof For a function $h \in \mathcal{S}^{*}(\varphi)$, in the paper [19, Corollary $\left.3^{\prime}\right]$ it is shown that

$$
\left|\arg \frac{h(z)}{z}\right| \leq \max _{|z|=r} \arg \frac{h_{\varphi}(z)}{z}, \quad|z|=r .
$$

The result then follows easily as the relation (3.3) holds.

Corollary 3.1 If $f \in \mathcal{S}_{b}^{\prime \prime}(\mu, A, B)$, then for $|z|=r$,

$$
\left|\arg \frac{f(z)^{\frac{1}{\mu}}}{(1-z)}\right| \leq \frac{A-B}{2 B} \max _{|z|=r} \arg (1+B z) \quad \text { for } B \neq 0
$$

and

$$
\left|\arg \frac{f(z)^{\frac{1}{\mu}}}{(1-z)}\right| \leq \frac{1}{2} \max _{|z|=r} \arg \exp (A z) \quad \text { for } B=0 .
$$

Corollary 3.2 Iff $\in \mathcal{S}_{b}^{*}(\mu, \beta)$, then for $|z|=r$

$$
\left|\arg \frac{f(z)^{\frac{1}{\mu}}}{(1-z)}\right| \leq(1-\beta) \max _{|z|=r} \arg \frac{1}{(1-z)} .
$$

Theorem 3.3 Let $\varphi(z)=z h_{\varphi}^{\prime}(z) / h_{\varphi}(z)$ and

$$
\min _{|z|=r}|\varphi(z)|=\varphi(-r) \quad \text { and } \quad \max _{|z|=r}|\varphi(z)|=\varphi(r) .
$$


Also, let

$$
H_{\varphi 1}=\frac{|\mu||1-z|^{\mu}}{2 r}\left(\frac{h_{\varphi}(-r)}{-r}\right)^{\frac{\mu}{2}}\left(-\left|\frac{1+z}{1-z}\right|+\varphi(-r)\right)
$$

and

$$
H_{\varphi 2}=\frac{|\mu||1-z|^{\mu}}{2 r}\left(\frac{h_{\varphi}(r)}{r}\right)^{\frac{\mu}{2}}\left(\left|\frac{1+z}{1-z}\right|+\varphi(r)\right) .
$$

For $\mu \in \mathbb{R}$, if $f \in \mathcal{S}_{b}^{*}(\mu, \varphi)$ then

$$
H_{\varphi 1} \leq\left|f^{\prime}(z)\right| \leq H_{\varphi 2} .
$$

Proof By Definition 1.2, for $f \in \mathcal{S}_{b}^{*}(\mu, \varphi)$, we have

$$
\frac{2}{\mu} \frac{z f^{\prime}(z)}{f(z)}+\frac{1+z}{1-z} \prec \varphi(z), \quad z \in \mathbb{D} .
$$

When (3.4) holds, the above subordination indicates that

$$
\varphi(-r) \leq\left|\frac{2}{\mu} \frac{z f^{\prime}(z)}{f(z)}+\frac{1+z}{1-z}\right| \leq \varphi(r), \quad|z|=r .
$$

This shows that

$$
-\left|\frac{1+z}{1-z}\right|+\varphi(-r) \leq\left|\frac{2}{\mu} \frac{z f^{\prime}(z)}{f(z)}\right| \leq\left|\frac{1+z}{1-z}\right|+\varphi(r)
$$

or

$$
\frac{|\mu|}{2 r}\left(-\left|\frac{1+z}{1-z}\right|+\varphi(-r)\right) \leq\left|\frac{f^{\prime}(z)}{f(z)}\right| \leq \frac{|\mu|}{2 r}\left(\left|\frac{1+z}{1-z}\right|+\varphi(r)\right) .
$$

For $\mu \in \mathbb{R}$, Theorem 3.1 gives

$$
|1-z|^{\mu}\left(\frac{h_{\varphi}(-r)}{-r}\right)^{\frac{\mu}{2}} \leq|f(z)| \leq|1-z|^{\mu}\left(\frac{h_{\varphi}(r)}{r}\right)^{\frac{\mu}{2}} .
$$

Combining (3.5) and (3.6), the desired results follows.

We have the following corollaries as (3.4) holds.

Corollary 3.3 Let $\varphi(z)=z h_{\varphi}^{\prime}(z) / h_{\varphi}(z)$. For $B \neq 0$, let

$$
H_{\varphi 1}=\frac{|\mu||1-z|^{\mu}}{2 r}(1-B r)^{\frac{\mu(A-B)}{2 B}}\left(-\left|\frac{1+z}{1-z}\right|+\frac{1-A r}{1-B r}\right)
$$

and

$$
H_{\varphi 2}=\frac{|\mu||1-z|^{\mu}}{2 r}(1+B r)^{\frac{\mu(A-B)}{2 B}}\left(\left|\frac{1+z}{1-z}\right|+\frac{1+A r}{1+B r}\right) .
$$


For $B=0$, let

$$
H_{\varphi 1}=\frac{|\mu||1-z|^{\mu}}{2 r} \exp \left(\frac{-\mu A r}{2}\right)\left(-\left|\frac{1+z}{1-z}\right|-r \exp (-A r)\right)
$$

and

$$
H_{\varphi 2}=\frac{|\mu||1-z|^{\mu}}{2 r} \exp \left(\frac{\mu A r}{2}\right)\left(\left|\frac{1+z}{1-z}\right|+r \exp (A r)\right) .
$$

For $\mu \in \mathbb{R}$, iff $\in \mathcal{S}_{b}^{*}(\mu, A, B)$ then

$$
H_{\varphi 1} \leq\left|f^{\prime}(z)\right| \leq H_{\varphi 2}
$$

Corollary 3.4 Let $\varphi(z)=z h_{\varphi}^{\prime}(z) / h_{\varphi}(z)$,

$$
H_{\varphi 1}=\frac{|\mu||1-z|^{\mu}}{2 r(1+r)^{\mu(1-\beta)}}\left(-\left|\frac{1+z}{1-z}\right|+\frac{1-(1-2 \beta) r}{1+r}\right)
$$

and

$$
H_{\varphi 2}=\frac{|\mu||1-z|^{\mu}}{2 r(1-r)^{\mu(1-\beta)}}\left(\left|\frac{1+z}{1-z}\right|+\frac{1+(1-2 \beta) r}{1-r}\right) .
$$

For $\mu \in \mathbb{R}$, iff $\in \mathcal{S}_{b}^{*}(\mu, \beta)$ then

$$
H_{\varphi 1} \leq\left|f^{\prime}(z)\right| \leq H_{\varphi 2}
$$

\section{Necessary and sufficient condition}

Theorem 4.1 Let $\varphi$ be a convex univalent function defined on $\mathbb{D}$. The function $f \in \mathcal{S}_{b}^{*}(\mu, \varphi)$ if and only iffor all $|s| \leq 1,|t| \leq 1$,

$$
\frac{s}{t}\left(\frac{1-t z}{1-s z}\right)^{2}\left(\frac{f(s z)}{f(t z)}\right)^{\frac{2}{\mu}} \prec \frac{h_{\varphi}(s z)}{h_{\varphi}(t z)}
$$

where $h_{\varphi}(z)=z \exp \left(\int_{0}^{z}((\varphi(\zeta)-1) / \zeta) d \zeta\right)$.

Proof Ruscheweyh [20, Theorem 1] showed that for $\varphi$ a convex univalent function, $F$ as in the hypothesis and $h \in \mathcal{A}$

$$
\frac{z h^{\prime}(z)}{h(z)} \prec \varphi(z)
$$

if and only if for all $|s| \leq 1,|t| \leq 1$,

$$
\frac{h(s z)}{h(t z)} \prec \frac{h_{\varphi}(s z)}{h_{\varphi}(t z)} .
$$


From the relation (3.3), we know that $f \in \mathcal{S}_{b}^{*}(\mu, \varphi)$ if and only if $h \in \mathcal{S}^{*}(\varphi)$. Substituting (3.2) in (4.1), we have

$$
\frac{\frac{s z}{(1-s z)^{2}} f(s z)^{\frac{2}{\mu}}}{\frac{t z}{(1-t z)^{2}} f(t z)^{\frac{2}{\mu}}} \prec \frac{h_{\varphi}(s z)}{h_{\varphi}(t z)}
$$

and hence the desired result follows.

The following corollaries hold for $\varphi(z)=\frac{1+A z}{1+B z}$ is convex univalent on $\mathbb{D}$.

Corollary 4.1 The function $f \in \mathcal{S}_{b}^{*}(\mu, A, B)$ if and only iffor all $|s| \leq 1,|t| \leq 1$,

$$
\begin{aligned}
& \left(\frac{1-t z}{1-s z}\right)^{\mu}\left(\frac{f(s z)}{f(t z)}\right) \prec\left(\frac{1+B s z}{1+B t z}\right)^{\frac{\mu(A-B)}{2 B}} \quad \text { for } B \neq 0, \\
& \left(\frac{1-t z}{1-s z}\right)^{\mu}\left(\frac{f(s z)}{f(t z)}\right) \prec \exp \left(\frac{\mu A z(s-t)}{2}\right) \quad \text { for } B=0 .
\end{aligned}
$$

Let $0 \leq \beta<1, A=1-2 \beta$ and $B=-1$ in Corollary 4.1 and hence we have the result.

Corollary 4.2 [18] The function $f \in \mathcal{S}_{b}^{*}(\mu, \beta)$ if and only iffor all $|s| \leq 1,|t| \leq 1$,

$$
\left(\frac{1-t z}{1-s z}\right)^{\mu} \frac{f(s z)}{f(t z)} \prec\left(\frac{1-t z}{1-s z}\right)^{\mu(1-\beta)}
$$

Theorem 4.2 as well as Corollaries 4.3 and 4.4 below are respectively special cases of Theorem 4.1 and Corollaries 4.1 and 4.2 when $s=1$ and $t=0$. However, we prove the below without the convexity assumption on $\varphi$.

Theorem 4.2 Iff $\in \mathcal{S}_{b}^{*}(\mu, \varphi)$, then

$$
\frac{f(z)^{\frac{2}{\mu}}}{(1-z)^{2}} \prec \frac{h_{\varphi}(z)}{z}
$$

where $h_{\varphi}(z)=z \exp \left(\int_{0}^{z}((\varphi(\zeta)-1) / \zeta) d \zeta\right)$.

Proof Clearly $z h_{\varphi}^{\prime}(z) / h_{\varphi}(z)=\varphi(z)$. If $h \in \mathcal{S}^{*}(\varphi)$, then

$$
\frac{z h^{\prime}(z)}{h(z)} \prec \frac{z h_{\varphi}^{\prime}(z)}{h_{\varphi}(z)} .
$$

Therefore by [19, Theorem $\left.1^{\prime}\right]$

$$
\frac{h(z)}{z} \prec \frac{h_{\varphi}(z)}{z} .
$$

Let $h(z)$ be defined as in (3.2) and hence we arrive at the desired conclusion. 
Corollary 4.3 Iff $\in \mathcal{S}_{b}^{*}(\mu, A, B)$ then

$$
\frac{f(z)}{(1-z)^{\mu}} \prec(1+B z)^{\frac{\mu(A-B)}{2 B}} \text { for } B \neq 0
$$

and

$$
\frac{f(z)}{(1-z)^{\mu}} \prec \exp \left(\frac{\mu A z}{2}\right) \quad \text { for } B=0 .
$$

When $0 \leq \beta<1, A=1-2 \beta$ and $B=-1$, the above corollary reduces to the following result.

Corollary 4.4 [18] Iff $\in \mathcal{S}_{b}^{*}(\mu, \beta)$ then

$$
\frac{f(z)}{(1-z)^{\mu}} \prec \frac{1}{(1-z)^{\mu(1-\beta)}} .
$$

\section{Coefficient estimate for $f \in \mathcal{S}_{b}^{*}(\varphi)$}

In particular, when $\mu=1,(1.2)$ becomes

$$
2 \frac{z f^{\prime}(z)}{f(z)}+\frac{1+z}{1-z} \prec \varphi(z), \quad z \in \mathbb{D} .
$$

We denote the class satisfying the above subordination as $\mathcal{S}_{b}^{\prime \prime}(\varphi)$.

Theorem 5.1 Let $\varphi(z)=1+B_{1} z+B_{2} z^{2}+\cdots$. If $f \in \mathcal{S}_{b}^{*}(\varphi)$, then the coefficients $d_{1}, d_{2}, d_{3}$ satisfy the following inequalities:

$$
\begin{aligned}
& \left|d_{1}\right| \leq \frac{B_{1}}{2}+1, \\
& \left|d_{2}\right| \leq \frac{B_{1}}{4} \max \left\{1,\left|\frac{B_{2}}{B_{1}}+\frac{B_{1}}{2}\right|\right\}+\frac{B_{1}}{2}, \\
& \left|d_{3}\right| \leq \frac{B_{1}}{6} H\left(\frac{6 B_{1}^{2}+16 B_{2}}{8 B_{1}}, \frac{B_{1}^{3}+6 B_{1} B_{2}+8 B_{3}}{8 B_{1}}\right)+\frac{B_{1}}{4} \max \left\{1,\left|\frac{B_{2}}{B_{1}}+\frac{B_{1}}{2}\right|\right\},
\end{aligned}
$$

where $H\left(q_{1}, q_{2}\right)^{\mathrm{a}}$ is as defined in [21] (see also [22, Lemma 3]) and

$$
\left|d_{2}-v d_{1}^{2}\right| \leq \begin{cases}\frac{B_{1}}{4}\left(\frac{B_{2}}{B_{1}}-(2 v-1) \frac{B_{1}}{2}\right)+(2 v+1) \frac{B_{1}}{2}+2 v, & v \leq \sigma_{1}, \\ \frac{B_{1}}{4}+(2 v+1) \frac{B_{1}}{2}+2 v, & \sigma_{1} \leq v \leq \sigma_{2}, \\ \frac{B_{1}}{4}\left((2 v-1) \frac{B_{1}}{2}-\frac{B_{2}}{B_{1}}\right)+(2 v+1) \frac{B_{1}}{2}+2 v, & v \geq \sigma_{2},\end{cases}
$$

where

$$
\sigma_{1}=\frac{1}{B_{1}}\left(\frac{B_{2}}{B_{1}}-1\right)+\frac{1}{2}, \quad \sigma_{2}=\frac{1}{B_{1}}\left(\frac{B_{2}}{B_{1}}+1\right)+\frac{1}{2} .
$$

Proof Define the function $g(z)=1+g_{1} z+g_{2} z^{2}+\cdots$ by

$$
g(z)=\frac{f(z)}{(1-z)}, \quad z \in \mathbb{D}
$$


Then a computation shows that

$$
2 \frac{z g^{\prime}(z)}{g(z)}+1=2 \frac{z f^{\prime}(z)}{f(z)}+\frac{1+z}{1-z}
$$

Since $f \in \mathcal{S}_{b}^{*}(\varphi)$, we have

$$
2 \frac{z g^{\prime}(z)}{g(z)}+1 \prec \varphi(z)
$$

or there is an analytic function $w(z)=w_{1} z+w_{2} z^{2}+\cdots$ such that

$$
2 \frac{z g^{\prime}(z)}{g(z)}+1=\varphi(w(z))
$$

Since

$$
2 \frac{z g^{\prime}(z)}{g(z)}+1=1+2 g_{1} z+\left(-2 g_{1}^{2}+4 g_{2}\right) z^{2}+\left(2 g_{1}^{3}-6 g_{1} g_{2}+6 g_{3}\right) z^{3}+\cdots
$$

and

$$
\varphi(w(z))=1+B_{1} w_{1} z+\left(B_{2} w_{1}^{2}+B_{1} w_{2}\right) z^{2}+\left(B_{3} w_{1}^{3}+2 B_{2} w_{1} w_{2}+b_{1} w_{3}\right) z^{3}+\cdots,
$$

we see that

$$
\begin{aligned}
& g_{1}=\frac{B_{1} w_{1}}{2}, \\
& g_{2}=\frac{B_{1}}{4}\left(w_{2}+\left(\frac{B_{2}}{B_{1}}+\frac{B_{1}}{2}\right) w_{1}^{2}\right), \\
& g_{3}=\frac{B_{1}}{6}\left(w_{3}+\left(\frac{6 B_{1}^{2}+16 B_{2}}{8 B_{1}}\right) w_{1} w_{2}+\left(\frac{B_{1}^{3}+6 B_{1} B_{2}+8 B_{3}}{8 B_{1}}\right) w_{1}^{3}\right) .
\end{aligned}
$$

In view of the well-known inequality $\left|w_{1}\right| \leq 1$, we have

$$
\left|g_{1}\right| \leq \frac{B_{1}}{2}
$$

Applying [23, inequality 7, p.10] and [22, Lemma 3] (see also [21]), we get

$$
\left|g_{2}\right| \leq \frac{B_{1}}{4} \max \left\{1,\left|\frac{B_{2}}{B_{1}}+\frac{B_{1}}{2}\right|\right\}
$$

and

$$
\left|g_{3}\right| \leq \frac{B_{1}}{6} H\left(\frac{6 B_{1}^{2}+16 B_{2}}{8 B_{1}}, \frac{B_{1}^{3}+6 B_{1} B_{2}+8 B_{3}}{8 B_{1}}\right),
$$

respectively. Also, we see that applying [22, Lemma 1] (see also [19]) to inequality

$$
g_{2}-v g_{1}^{2}=\frac{B_{1}}{4}\left(w_{2}-\left((2 v-1) \frac{B_{1}}{2}-\frac{B_{2}}{B_{1}}\right) w_{1}^{2}\right)
$$


yields

$$
\left|g_{2}-v g_{1}^{2}\right| \leq \begin{cases}\frac{B_{1}}{4}\left(\frac{B_{2}}{B_{1}}-(2 v-1) \frac{B_{1}}{2}\right), & v \leq \sigma_{1}, \\ \frac{B_{1}}{4}, & \sigma_{1} \leq v \leq \sigma_{2}, \\ \frac{B_{1}}{4}\left((2 v-1) \frac{B_{1}}{2}-\frac{B_{2}}{B_{1}}\right), & v \geq \sigma_{2}\end{cases}
$$

for $\sigma_{1}$ and $\sigma_{2}$ as in the hypothesis. Todorov in [3] shows that for

$$
g(z)=1+\sum_{1}^{\infty} g_{n} z^{n}
$$

the coefficient

$$
g_{n}=1+d_{1}+d_{2}+\cdots+d_{n}
$$

and hence from the above relation the desired results are obtained.

Corollary 5.1 When $\varphi(z)=(1+z) /(1-z)$, our results coincide with [3, Corollary 2.3].

Remark 5.1 All the results for the special case when $\mu=1$ or the class starlike with respect to a boundary point defined by subordination were presented at the 8th International Symposium on GFTA, 27-31 August 2012, Ohrid, Republic of Macedonia and thereafter published as [24].

\section{Competing interests}

The authors declare that they have no competing interests.

\section{Authors' contributions}

The first author MHM is currently a PhD student under supervision of the second author MD and jointly worked on deriving the results. All authors read and approved the final manuscript.

\section{Acknowledgements}

The work here is partially supported by MOHE:LRGS/TD/2011/UKM/ICT/03/02.

\section{Endnote}

a The expression for $H$ is too lengthy to be reproduced here. See [21] or [22] for the full expression.

Received: 29 December 2012 Accepted: 1 May 2013 Published: 31 May 2013

\section{References}

1. Robertson, MS: Univalent functions starlike with respect to a boundary point. J. Math. Anal. Appl. 81(2), 327-345 (1981)

2. Lyzzaik, A: On a conjecture of M.S. Robertson. Proc. Am. Math. Soc. 91(1), 108-110 (1984)

3. Todorov, PG: On the univalent functions starlike with respect to a boundary point. Proc. Am. Math. Soc. 97(4), 602-604 (1986)

4. Silverman, H, Silvia, EM: Subclasses of univalent functions starlike with respect to a boundary point. Houst. J. Math. 16(2), 289-299 (1990)

5. Abdullah, AS, Ali, RM, Singh, V: On functions starlike with respect to a boundary point. Ann. Univ. Mariae Curie-SkTodowska, Sect. A 50, 7-15 (1996)

6. Chên, MP, Owa, S: Generalization of Robertson functions. In: Complex Analysis and Its Applications (Hong Kong, 1993). Pitman Res. Notes Math. Ser., vol. 305, pp. 159-165. Longman, Harlow (1994)

7. Dong, J, Liu, L: Distortion properties of a class of functions starlike with respect of a boundary point. Heilongjiang Daxue Ziran Kexue Xuebao 15(4), 1-6 (1998)

8. Elin, M, Reich, S, Shoikhet, D: Dynamics of inequalities in geometric function theory. J. Inequal. Appl. 6(6), 651-664 (2001)

9. Lecko, A: On the class of functions starlike with respect to a boundary point. J. Math. Anal. Appl. 261 (2), 649-664 (2001) 
10. Lecko, A, Lyzzaik, A: A note on univalent functions starlike with respect to a boundary point. J. Math. Anal. Appl. 282(2), 846-851 (2003)

11. Xu, Q-H, Lv, C-B, Srivastava, HM: Coefficient estimates for the inverses of a certain general class of spirallike functions. Appl. Math. Comput. 219(12), 7000-7011 (2013)

12. Elin, M, Reich, S, Shoikhet, D: Holomorphically accretive mappings and spiral-shaped functions of proper contractions. Nonlinear Anal. Forum 5, 149-161 (2000)

13. Elin, M, Shoikhet, D: Angle distortion theorems for starlike and spirallike functions with respect to a boundary point. Int. J. Math. Math. Sci. 2006, Art. ID 81615 (2006)

14. Lecko, A: The class of functions spirallike with respect to a boundary point. Int. J. Math. Math. Sci. 2004(37-40), 2133-2143 (2004)

15. Shoikhet, D: Representations of holomorphic generators and distortion theorems for spirallike functions with respect to a boundary point. Int. J. Pure Appl. Math. 5(3), 335-361 (2003)

16. Aharonov, D, Elin, M, Shoikhet, D: Spiral-like functions with respect to a boundary point. J. Math. Anal. Appl. 280(1), $17-29(2003)$

17. Elin, M, et al.: Dynamics of spirallike functions. In: Complex Analysis and Dynamical Systems. Contemp. Math., vol. 364, pp. 41-57. Am. Math. Soc., Providence (2004)

18. Elin, M: Covering and distortion theorems for spirallike functions with respect to a boundary point. Int. J. Pure Appl. Math. 28(3), 387-400 (2006)

19. Ma, WC, Minda, D: A unified treatment of some special classes of univalent functions. In: Proceedings of the Conference on Complex Analysis (Tianjin, 1992). Conf. Proc. Lecture Notes Anal., vol. I, pp. 157-169. Int. Press, Cambridge (1994)

20. Ruscheweyh, S: A subordination theorem for F-like functions. J. Lond. Math. Soc. 13(2), 275-280 (1976)

21. Prokhorov, DV, Szynal, J: Inverse coefficients for $(\alpha, \beta)$-convex functions. Ann. Univ. Mariae Curie-Skīodowska, Sect. A 35(1981), 125-143 (1984)

22. Ali, RM, Ravichandran, V, Seenivasagan, N: Coefficient bounds for $p$-valent functions. Appl. Math. Comput. 187(1), 35-46 (2007)

23. Keogh, FR, Merkes, EP: A coefficient inequality for certain classes of analytic functions. Proc. Am. Math. Soc. 20, 8-12 (1969). MR0232926

24. Mohd, MH, Darus, M: Starlike function with respect to a boundary point defined by subordination. Adv. Math. Sci. J. 1(1), 15-21 (2012)

doi:10.1186/1029-242X-2013-274

Cite this article as: Haji Mohd and Darus: On a class of spiral-like functions with respect to a boundary point related to subordination. Journal of Inequalities and Applications 2013 2013:274.

\section{Submit your manuscript to a SpringerOpen ${ }^{\ominus}$ journal and benefit from:}

- Convenient online submission

- Rigorous peer review

Immediate publication on acceptance

- Open access: articles freely available online

- High visibility within the field

- Retaining the copyright to your article 